\title{
La inteligencia artificial como prueba científica en el proceso penal español
}

\author{
Artificial intelligence as scientific evidence \\ in the Spanish criminal process
}

\author{
Miren Josune Pérez Estrada ${ }^{1}$ \\ Universidad del País Vasco, Leioa, Bizkaia, España \\ mirenjosune.perez@ehu.eus \\ http://orcid.org/0000-0001-7402-4863
}

\begin{abstract}
Resumen: En este trabajo se reflexiona sobre el posible uso de la inteligencia artificial en el proceso penal como ayuda en la actividad jurisdiccional probatoria. Para llevar a cabo este análisis se realizará, en primer lugar, una configuración de la herramienta de inteligencia artificial, continuaremos con la delimitación de la prueba científica en el proceso penal español y, finalizaremos, concluyendo sobre la posibilidad de utilizar la inteligencia artificial como prueba científica en el proceso penal o la posibilidad de usarse con el resto de medios de prueba tradicionales de manera que los haga más efectivos y le sirva al juez de ayuda en la función de valoración de la prueba.
\end{abstract}

PALABRAS ClaVE: Inteligencia artificial; proceso penal; prueba científica; reconocimiento facial; garantías procesales.

\begin{abstract}
This work analyses the use of artificial intelligence in criminal proceedings, not from its application to the resolution of legal disputes, but as an aid in probative judicial activity. To carry out this research, a configuration of the artificial intelligence tool will be carried out first, we will continue with the delimitation of the scientific test in the Spanish criminal process and conclude on the possibility of using artificial intelligence as scientific evidence.
\end{abstract}

KEYwORDs: Artificial intelligence; criminal proceedings; scientific evidence; procedural safeguards.

1 Profesora Adjunta de Derecho Procesal (acreditada Agregada). Facultad de Derecho. Sección Bizkaia, Universidad del País Vasco. Doctora en Derecho. 
SUMARIO: I. Introducción. II. Configuración de la Inteligencia Artificial. 1. Contextualización de la Inteligencia Artificial. 2. Concepto y funcionamiento de la Inteligencia Artificial. 2.1. Concepto de Inteligencia Artificial. 2.2. Funcionamiento de la Inteligencia Artificial. III. La Inteligencia Artificial en el proceso penal español como prueba científica. 1. Concepto y naturaleza de la prueba científica. 2. La aplicación de la Inteligencia Artificial en el proceso penal. 2.1. Tecnología de reconocimiento facial. 2.2. El sentido de la inteligencia artificial como prueba científica en el proceso penal. IV. Conclusiones. V. Referencias bibliográficas.

\section{INTRODUCCIÓN²}

En los últimos años la inteligencia artificial se ha introducido en todos los ámbitos de nuestra vida. A menudo utilizamos herramientas de inteligencia artificial para muy diferentes usos, en la rutina diaria, el sector industrial o el ámbito sanitario. Incluso los servicios públicos se están beneficiando de las soluciones eficientes que ofrece la inteligencia artificial, como se señala en el Plan Coordinado sobre la Inteligencia Artificial de la Comisión Europea, en el apartado I dedicado a la "Introducción- La estrategia europea en materia de inteligencia artificial"

De esta transformación no ha quedado al margen el Derecho. El uso de la inteligencia artificial y, en concreto, la utilización de algoritmos en el Derecho privado es ya una realidad ${ }^{4}$. Existen aplicaciones dirigidas

2 Este trabajo se ha realizado en el contexto de las actividades que realiza el Grupo de investigación consolidado "Derechos Fundamentales y Unión Europea. Especial referencia al Espacio de Libertad, Seguridad y Justicia de la Unión Europea" (GIC IT-673-13, 2019-2021), así como dentro del contexto del Proyecto H2020-SWAFS17/01.

3 Comunicación de la Comisión al Parlamento Europeo, al Consejo Europeo, al Consejo, al Comité Económico y Social Europeo y al Comité de las Regiones, de fecha 7.12.2018, COM (2018) 795 final, sobre el "Plan coordinado sobre la inteligencia artificial", p. 2.

4 De referencia en la materia HARTUNG, Markus, BUES, Micha-Manuel y HALBLEIB, Gernot, AAVV, Legal Tech. A Practitioner's Guide, Germany: Beck/Hart, 2018. En la obra se realiza un análisis exhaustivo del estado actual 
a los operadores jurídicos que, o bien, estudian las decisiones judiciales con un fin de justicia predictiva o elaboran perfiles de jueces tomando como base su actividad jurisdiccional; hay, también, herramientas dirigidas a la búsqueda y procesamiento de información jurídica ${ }^{5}$. En el ámbito jurisdiccional, empiezan a aparecer sistemas automatizados de toma de decisiones basados en algoritmos que persiguen la rapidez y certeza jurídica en la aplicación del derecho, la seguridad en el resultado; en definitiva, el acierto en la decisión judicial. Como consecuencia, en la actualidad, en determinados sistemas penales, se usan algoritmos predictivos que ayudan al juez en la toma de determinadas decisiones judiciales con la idea de lograr eficacia en el proceso, de racionalizar la Justicia ${ }^{6}$. Si bien, este tipo de mecanismos inteligentes no están exentos de polémica con motivo de los sesgos algorítmicos que contienen, por lo que su tratamiento

y futuro de la LegalTech a nivel mundial. Estudia la aplicación de la tecnología jurídica y explica cómo y en qué medida se está utilizando el trabajo automatizado y la inteligencia artificial.

5 Un repaso por las actuales herramientas de inteligencia artificial, LACRUZ MANTECÓN, Miguel L., "Cibernética y Derecho Europeo: ¿una inteligencia robótica?". Madrid: Diario La Ley, n. 9376, Sección Doctrina, 2019, nos recuerda la evolución de esta materia desde la Jurimetría, basada en el análisis y previsión de sentencias futuras, hacia el uso de la inteligencia artificial en la aplicación del Derecho. MAGRO SERVET, Vicente. La aplicación de la inteligencia artificial en la Administración de Justicia. Madrid: Diario La Ley, n.. 9268, Sección Doctrina, 2018.

6 Esta idea de certeza jurídica aboca hacia sistemas artificiales inteligentes ante la imposibilidad de conseguir seguridad jurídica en las decisiones judiciales. Como pone de manifiesto GUZMÁN FLUJA, Vicente C., Sobre la aplicación de la inteligencia artificial a la solución de conflictos (Reflexiones acerca de una transformación tan apasionante como compleja. In: BARONA VILAR, Silvia (ed.). Justicia Civil y Penal en la era global, Valencia: Tirant lo Blanch, , 2017, p. 68, “...Desde hace ya bastantes años se ha venido especulando con la posibilidad de que la actividad de decisión jurídica de los conflictos pudiera ser llevada a cabo por un robot. Hablamos del juez-robot entendido como sustituto pleno del juez humano. Estamos más cerca de ello, sin duda, ...pero lo que ha llegado con fuerza es la capacidad de predecir el resultado de un litigio, lo que se llama predictibilidad de la decisión judicial, entendida como un sistema de ayuda a la toma de decisiones, no sólo del Juez, ni siquiera principalmente, sino sobre todo de los litigantes". También BARONA VILAR, Silvia. Cuarta revolución industrial (4.0.) o ciberindustria en el proceso penal: revolución digital, inteligencia artificial y el camino hacia la robotización de la justicia. Revista Jurídica Digital UANDES, Vol. 3, n. 1, Chile, 2019, pp. 1-17. 
jurídico debe realizarse desde el respeto a las normas fundamentales y de derechos humanos.

En este trabajo se pretende analizar la utilización de la inteligencia artificial en el proceso penal como ayuda en la actividad jurisdiccional probatoria ${ }^{7}$. Para llevar a cabo esta investigación se realizará, en primer lugar, una configuración de la herramienta de inteligencia artificial, continuaremos con la delimitación de la prueba científica en el proceso penal español y finalizaremos concluyendo sobre la posibilidad de usar la inteligencia artificial como prueba científica.

\section{Configuración de la Inteligencia Artificial}

\section{Contextualización de la Inteligencia Artificial}

Se empieza a cuestionar por la doctrina más especializada el encaje de la inteligencia artificial dentro del contexto de la llamada cuarta revolución industrial ${ }^{8}$. Se considera que es más apropiado el calificativo "convergencia" tanto para hablar de las diferentes tecnologías digitales como para describir la interacción que surge entre ellas ${ }^{9}$. Es más conveniente hablar de transversalidad en el uso de esta tecnología digital que, además, potencia sus efectos cuando se interrelaciona.

7 PÉREZ ESTRADA, M. Josune. El uso de algoritmos en el proceso penal y el derecho a un proceso con todas las garantías. In: BARONA VILAR, Silvia (ed.). Claves de la justicia penal: feminización, inteligencia artificial, supranacionalidad y seguridad. Valencia: Tirant lo Blanch, 2019, pp. 235-254. DE MIGUEL BERIAIN, Iñigo; PÉREZ ESTRADA, M. Josune. La inteligencia artificial en el proceso penal español: un análisis de su admisibilidad sobre la base de los derechos fundamentales implicados, Revista de derecho UNED (RDUNED), n 25, Madrid, 2019, pp. 531-561. https://doi.org/10.5944/ rduned.25.2019.27013

8 QUADRA-SALCEDO FERNÁNDEZ DEL CASTILLO, Tomás, Derechos fundamentales, democracia y mercado en la edad digital, Derecho Digital e Innovación, núm. 1, Madrid, enero-marzo 2019, p. 2,

9 Las tecnologías “convergentes" o "emergentes" se identifican por las iniciales NBIC (nano, bio, info...) que corresponde con nanotecnología, biomedicina, informática y neurociencia. BONAZZI, M., "Reconstructing man? The power of converging technologies", 2006. http://cordis.europa.eu/wire/index. cfm?fuseaction=article. Detail \&rcn=11117 (fecha acceso: $20 / 05 / 2021)$ 
La idea de superar el concepto de revolución industrial hacia el de "convergencia tecnológica" radica, básicamente, en la afectación que supone. Nos referimos al hecho de que si la revolución industrial supuso un cambio radical en el modo de producción, la tecnología convergente supone un cambio en la propia esencia del ser humano, que puede llegar incluso modificar la conducta de la persona ${ }^{10} \mathrm{y}$ afectar a sus derechos fundamentales ${ }^{11}$.

Se espera que la inteligencia artificial tenga un desarrollo exponencial en los próximos años; precisamente, porque uno de los factores de esa rápida evolución es la convergencia con el resto de tecnologias emergentes, que avanzan velozmente. Esta evolución tecnológica provoca consecuencias en la propia configuración de la persona, por lo que va a ser necesario que se proteja la esencia personal desde el sistema de los derechos fundamentales. No obstante, no hay que olvidar, tampoco, los beneficios que puede tener para la evolución de la sociedad y del Derecho el uso de estas tecnologías. Por lo tanto, se debe ir en esa doble dirección, aprovechar los beneficios que pueden proporcionan estas tecnologías y, en este caso, valorar su aplicación en el ámbito probatorio del proceso penal, aunque preservando, eso sí las garantías procesales fundamentales que rigen este proceso.

10 PASTOR GARCÍA, Luis Miguel; GARCÍA CUADRADO, José Ángel. Modernity and postmodernity in the genesis of posthumanism, Cuadernos de bioética, (Ejemplar dedicado a: Posthumano, más que humano), Vol. 25, $\mathrm{n}^{\circ} 85,2014$, pp. 335-350, concluyen que estas corrientes niegan la esencia de la persona e intentan construir algo diferente a ella en el futuro.

11 QUADRA-SALCEDO FERNÁNDEZ DEL CASTILLO, Tomás. Retos, riesgos y oportunidades de la Sociedad digital". In: QUADRA-SALCEDO y PIÑAR MAÑAS (dir.), Sociedad Digital y Derecho. Madrid: BOE. Ministerio de Industria, Comercio y Turismo y Red.es, 2018, pp. 21 y ss., se ocupan de la protección de los derechos fundamentales, como la intimidad o el honor del individuo, al percatarse que las nuevas tecnologías ocasionan riesgos por sí mismas, de manera independiente al ser humano. Y lo que es más importante, mantienen la idea de que la protección de determinados derechos fundamentales del individuo cede ante la protección de la sociedad que se debe otorgar a la sociedad. 


\section{Concepto y funcionamiento de la Inteligencia Artificial}

\subsection{Concepto de Inteligencia Artificial}

Como acabamos de señalar es significativo el crecimiento que ha experimentado esta área científica en los últimos tiempos y existe una previsión de crecimiento exponencial de la inteligencia artificial en los próximos años ${ }^{12}$. No cabe duda, estamos ante una de las tecnologías que va a transformar la sociedad en el modo, en el que la conocemos, si no lo está haciendo $\mathrm{ya}^{13}$. Sin embargo, a pesar de esta creciente importancia no existe un término unívoco para definir la inteligencia artificial, sino muchos y provenientes de muy diversos sectores, en función de las características que presenta cada uno de ellos.

Esta falta de concepto único se pone de manifiesto por el propio Comité Económico y Social Europeo, en el "Dictamen sobre la inteligencia artificial: las consecuencias de la inteligencia artificial para el mercado único (digital), la producción, el consumo, el empleo y la sociedad" que recoge, en la Conclusión 2.1, que no existe una definición concreta y aceptada de la inteligencia artificial ${ }^{14}$. Por utilizar un concepto que nos pueda servir en el presente trabajo nos quedaremos con el que usa la Comisión Europea en el documento sobre "Inteligencia artificial para Europa"15: "El término «inteligencia artificial» se aplica a los sistemas que manifiestan un comportamiento inteligente, pues son capaces de analizar su entorno y pasar a la acción -con cierto grado de autonomía- con el fin

12 Incluso sin que la inteligencia artificial avanzara al ritmo que lo hace en la actualidad las aportaciones e implicaciones, en definitiva, el potencial de afectación en la vida diaria es significativo, apuntando sobremanera a cuatro grandes áreas entre las que destaca la justicia penal. De esta manera se expone en el Foro "Artificial intelligence. Emerging Opportunities, Challenges, and Implications for Policy and Research", United States Government Accountability Office, March 2018, p. 2.

13 Entre sus beneficios destacan un mayor crecimiento económico y progreso social como se señala en "Preparing for the future of artificial intelligence", Executive Office of the President National Science \& Technology Council Committee on Technology, 2016, pp. 3-4.

14 Diario Oficial de la Unión Europea (DOUE) de 31/08/2017.

15 Comunicación de la Comisión, Bruselas, 25.4.2018 COM (2018) 237 final, p. 1. 
de alcanzar objetivos específicos." Se trata de un concepto que pone el punto de mira en la potencialidad de los sistemas, en las aplicaciones de inteligencia artificial. Nos interesa en este trabajo la inteligencia artificial aplicada al proceso penal y, en concreto, la inteligencia artificial en la fase probatoria.

Aunque la inteligencia artificial aún no ha alcanzado un pleno desarrollo ${ }^{16}$ su gran potencial nos proporciona herramientas increíbles. Es por ello que deberíamos valorar la posibilidad de utilizar estas herramientas inteligentes en la fase probatoria del proceso penal. Antes de examinar la cuestión, vamos a analizar los dos grandes pilares en los que se basa el funcionamiento de la inteligencia artificial, nos referimos a la gran cantidad de datos que gestiona mediante el uso de algoritmos.

\subsection{Funcionamiento de la Inteligencia Artificial}

No es lo mismo inteligencia artificial que algoritmo. La inteligencia artificial es la inteligencia que muestran las máquinas cuando imitan las funciones "cognitivas" de los seres humanos, por ejemplo, al resolver problemas. Como hemos señalado se trata de un concepto aún en desarrollo. Sin embargo, lo que se ha generalizado son los sistemas automatizados de toma de decisiones basados en algoritmos ${ }^{17}$.

Los algoritmos son una serie de ecuaciones matemáticas que se entrelazan para proporcionar un resultado. Atienden a una instrucción concreta que sirve para solucionar un problema planteado previamente. Para lograrlo utiliza una gran base de datos ordenada, lo que se denomina Smart Data, en la que elige los datos, de manera aleatoria, con la finalidad

16 Algunos autores reconocen la dificultad de conocer el futuro desarrollo de la inteligencia artificial, como MÜULLER, Vincent C., BOSTROM, Nick. Future Progress in Artificial Intelligence: A Survey of Expert Opinion. In: MÜLLER, Vincent C. (ed.). Fundamental Issues of Artificial Intelligence., Berlin: Springer, 2014.

17 O’NEIL, Cathy Armas de destrucción matemática., Madrid: Capitán Swing, 2017. EDWARS, Llilian, VEALE, Michael. Slave to the algorithm? Why a "Right to an explanation" is probably not the remedy you are looking for. Drurham: Duke Law \& Technology Review, 2017, pp. 19-20, muestran una dependencia absoluta de nuestra sociedad actual hacia los algoritmos. 
de anticipar un comportamiento. La finalidad del algoritmo no es certificar la veracidad de una hipótesis planteada sino la búsqueda de correlaciones deterministas entre unos datos y otros ${ }^{18}$.

Los algoritmos, en modo alguno, son nuevos y se han aplicado en los programas informáticos desde décadas ${ }^{19}$. Su importancia actual radica en que han evolucionado de sistemas expertos, en los que los programadores trasladaban al algoritmo las normas para tomar las decisiones, a algoritmos que permiten el aprendizaje automático de la máquina ("machine learning"). Estos algoritmos de aprendizaje son más avanzados, más inteligentes y se muestran más eficaces en cuanto a su capacidad de anticipar comportamientos, pero tienen el problema de que su funcionamiento resulta incomprensible, lo que dificulta la legitimidad de las decisiones que adoptan ${ }^{20}$. Su creación parte de un proceso no sólo estadístico o matemático, sino complejo y humano que incluye recopilación, preparación y análisis de datos en diversas etapas, lo que no está exento de polémica porque en ese proceso, es evidente e inevitable, que se contengan ideologías propias de los creadores y diseñadores de los algoritmos ${ }^{21}$.

El desarrollo de la inteligencia artificial está íntimamente relacionado con el uso o con la disponibilidad de una gran cantidad de datos que son procesados por algoritmos ${ }^{22}$. Nos estamos refiriendo al

18 COTINO HUESO, Lorenzo. Big data e inteligencia artificial. Una aproximación a su tratamiento jurídico desde los derechos fundamentales. Madrid: Dilemata, 2017, pp. 24, 131-150.

19 Como nos lo recuerdan COGLIANESE; Cary and LEHR, David. Regulating by Robot: Administrative Decision Making in the Machine-Learning Era. Georgetown Law Journal, Forthcoming; U of Penn: Inst for Law \& Econ Research Paper, n. 17-8, febrero 2017, p. 3.

20 Así, la lógica de un algoritmo puede ser incomprensible para las personas. MITTELSTADT, Brent Daniel; ALLO, Patrick; TADDEO, Maria Rosaria; WATCHER, Sandra, FLORIDI, Luciano,.The ethics of algorithms: Mapping the debate" Oxford Internet Institute, Diciembre, 2016.

21 FRA, BigData: Discrimination in data-supported decision making, European Union Agency for Fundamental Rights, Viena, 2018, p. 4.

22 Recordemos la idea de manejar los datos ("playing with the data") y ejecutar el modelo ("running model"); expresiones que utilizan LEHR, David \& OHM, Paul. Playing with the Data: What Legal Scholars Should Learn About Machine Learning. Georgetown Law Journal, 2017. 
Big Data ${ }^{23}$ o, también, a la expresión "macrodatos", que es utilizada por el Parlamento Europeo, y que lo define como "la recopilación, análisis y acumulación constante de grandes cantidades de datos, incluidos datos personales, procedentes de diferentes fuentes y objeto de un tratamiento automatizado mediante algoritmos informáticos y avanzadas técnicas de tratamiento de datos, utilizando tanto datos almacenados como datos transmitidos en flujo continuo, con el fin de generar correlaciones, tendencias y patrones (analítica de macrodatos)" ${ }^{24}$.

Se trata de tecnología disruptiva que se incardina en la categoría más amplía de "tecnologías emergentes o convergentes". Una nueva tecnología de tratamiento y análisis de datos, un nuevo método de procesamiento de información" 25 pero con una característica fundamental, el procesamiento de la información se realiza a nivel global ${ }^{26}$. Como consecuencia de este tratamiento de datos masivos se infieren conclusiones imperceptibles que otra técnica de análisis de datos no es capaz de apreciar, lo que proporciona un conocimiento superior ${ }^{27}$. Incluso, la técnica Big Data

23 El uso del Big Data supone un uso global de los datos lo que, a su vez, conlleva una vigilancia global; pero su gran problema, entiendo, está, precisamente, en la percepción equivocada que se tiene de la libertad. El individuo sometido al Big Data no concibe una pérdida de libertad como tal. Como afirma BYUNGCHUL, Han. La sociedad de la transparencia. Barcelona: Herder, 2013, pp. 88 y 89 , el panóptico digital, que es el Big Data funciona sin ninguna óptica perspectivista y, por lo tanto, la vigilancia se puede producir desde todas las partes; creen que gozan de libertad.

24 Resolución de 14 de marzo de 2017, sobre las implicaciones de los "macrodatos" en los derechos fundamentales: privacidad, protección de datos, no discriminación, seguridad y aplicación de la ley (2016/2225(INI)).

25 MORENTE PARRA, Vanesa. Big Data o el arte de analizar datos masivos. Una reflexión crítica desde los derechos fundamentales. Derechos y Libertades, n. 41, Época II, junio 2019, p. 227.

26 MILÓN BELTRÁN, Noemí. "Retos para la privacidad en la Era Digital. Análisis económico y filosófico político del capitalismo contemporáneo”. Sociología y Tecnociencia. Revista digital de sociología del sistema tecnocientífico, vol. 2, n.. 5, 2015, p. 33.

27 BOYD, David; CRAWFORD, Kate. Critical questions for Big Data. Provocations for a cultural, technological and scholarly phenomenon., Journal Information, Communication and Society, n.. 5, vol. 15, 2012, pp. 662 y 663, hablan de "inteligencia superior" para describir la potencialidad de esta nueva tecnología en el tratamiento de los datos que es capaz de adelantar acontecimientos imposibles de detectar de otra forma. 
mediante el uso de algoritmos puede no sólo predecir acontecimientos futuros sino también que las máquinas tomen decisiones autónomas sin necesidad de que intervenga una persona ${ }^{28}$.

Precisamente, es en los datos, como la fuente que utilizan los algoritmos, donde es necesario poner especial atención porque va a ser la calidad de los datos en los que se basa el algoritmo los que van a conseguir que los resultados que se proporcionen tengan legitimidad. ${ }^{29}$

\section{La Inteligencia Artificial en el proceso penal español COMO PRUEBA CIENTÍFICA}

\section{Concepto y naturaleza de la prueba Científica}

Se plantea en este apartado la posibilidad de que se pueda utilizar esta tecnología inteligente en el proceso penal. En concreto, si es posible que la inteligencia artificial pueda suponer una mejora en la actividad probatoria ${ }^{30}$ que ayude a los medios de prueba tradicionales

28 COTINO HUESO, Lorenzo, "Big data e inteligencia artificial. Una aproximación a su tratamiento jurídico desde los derechos fundamentales", op. cit., p. 132.

29 SURDEN, Harry. Values Embedded in Legal Artificial Intelligence. Documento de investigación de estudios legales de la U. of Colorado, n. 17-17, 2017. Para SURDEN al margen del "manto de objetividad de la tecnología", los sistemas tecnológicos pueden tener valores sociales "incrustados" o embebidos en su diseño y que éstos sean contrarios a la igualdad, principios constitucionales y derechos humanos.

El Parlamento Europeo, en su Resolución de 14 de marzo de 2017, sobre las implicaciones de los macrodatos en los derechos fundamentales: privacidad, protección de datos, no discriminación, seguridad y aplicación de la ley (2016/2225(INI), Considerando m) pone de manifiesto que la "baja calidad" de los datos o los procedimientos "podrían dar lugar a algoritmos sesgados, correlaciones falsas, errores, una subestimación de las repercusiones éticas, sociales y legales, el riesgo de utilización de los datos con fines discriminatorios o fraudulentos y la marginación del papel de los seres humanos en esos procesos, lo que puede traducirse en procedimientos deficientes de toma de decisiones con repercusiones negativas en las vidas y oportunidades de los ciudadanos, en particular los grupos marginalizados, así como generar un impacto negativo en las sociedades y empresas".

30 Sobre derecho probatorio, prueba científica y pericial podemos consultar ASENSIO MELLADO, José María. "La prueba penal”. In: ASENSIO 
en la identificación del presunto autor del hecho o en la averiguación del hecho delictivo.

Aunque la tecnología que se está desarrollando podría tener aplicación en el proceso penal, no existe en derecho procesal una definición unívoca del concepto de prueba científica y las definiciones que se han dado, tanto por la doctrina como por la jurisprudencia, son incompletas. A pesar de ello, no se discute y se entiende sin problemas que la prueba científica se dirige a averiguar o probar hechos que necesitan conocimientos profesionales y técnicos muy avanzados tecnológicamente ${ }^{31}$.

No hay tampoco acuerdo por parte de la doctrina para concretar las diferentes ciencias o tecnologías que pueden comprender la prueba científica pero lo que sí es evidente es que muchos hechos criminales graves sólo se pueden descubrir o probar a través de tecnologías de nueva generación que faciliten la convicción judicial ${ }^{32}$.

Ni el legislador procesal ofrece un concepto de prueba pericial ni, menos aún, de prueba científica como una variedad de ésta. La única cuestión que regula el derecho procesal es cuándo procede realizar la

MELLADO, José María (dir.); FUENTES SORIANO, Olga (coord.). Derecho procesal penal (dir., coord.), Valencia: Tirant lo Blanch, 2020. PICÓ i JUNOY, Joan (dir.); ANDINO LÓPEZ, Juan Antonio (coord..); CERRATO GURI, Elisabet (coord.). La prueba pericial a examen. Madrid: J. M. Bosch Editor 2020. FERRER BELTRÁN, Jordi. Prueba y verdad en el derecho. Madrid: Marcial Pons, 2005.

31 Desarrolla este tema GÓMEZ COLOMER, Juan Luis. Los retos del proceso penal ante las nuevas pruebas que requieren tecnología avanzada: El análisis de ADN." In: GÓMEZ COLOMER, Juan Luis (coord.), La prueba de ADN en el proceso penal. Valencia: Tirant lo Blanch, 2014, pp. 23 y ss. PÉREZ GIL, Julio. El conocimiento científico en el proceso civil. Ciencia y tecnología en tela de juicio. Valencia: Tirant lo Blanch, 2010, pp. 42 a 47. GASCÓN ABELLÁN, Marina. Validez y valor de las pruebas científicas: la prueba del ADN. Universidad de Castilla- La Mancha, 2015. También coincide con el concepto que utiliza nuestra jurisprudencia habitualmente. Véanse, entre otras: STS (Sección $1^{\text {a }}$ ) 513/2010 de 2 de junio; STS (Sección $1^{\text {a }}$ ), 1069/2012 de 2 de diciembre; STS (Sección $1^{\mathrm{a}}$ ), 925/2012 de 8 de noviembre; STS (Sección $1^{\mathrm{a}}$ ), 191/2013 de 6 de marzo. STS (Sección $1^{\mathrm{a}}$ ), 128/2009 de 11 de febrero; STS (Sección $1^{\mathrm{a}}$ ) 922/2010 de 28 de octubre; STS (Sección $1^{\mathrm{a}}$ ), 540/2014 de 1 de julio, etc.

32 TARUFFO, Michele. La prueba de los hechos (trad. Ferrer Beltrán). Madrid: Trotta, 2002, p. 331, mantiene que no es posible obtener ninguna definición aceptable de prueba científica. 
práctica de prueba pericial en el proceso penal, ie., el objeto y finalidad de la prueba pericial. Así el art. 456 Ley de Enjuiciamiento Criminal (LECR) entiende que procede dicha prueba "Cuando sean necesarios conocimientos científicos, artísticos, técnicos o prácticos para valorar hechos o circunstancias relevantes en el asunto o adquirir certeza sobre ellos...”.

Ante la falta de un concreto concepto sobre lo que debe entenderse por prueba pericial científica unos determinados criterios o requisitos de fiabilidad científica, como existe en otros países $^{33}$, podrían ayudarnos a entender que una prueba tiene la categoría de científica y, por lo tanto, puede utilizarse para probar científicamente un hecho en un proceso, civil o penal" 34 .

Lo referente a la cuestión de la naturaleza jurídica de la prueba científica tampoco resulta ser un debate doctrinal pacífico. Si bien resultó superada la cuestión de considerar la prueba científica como prueba documental, debido en parte a la regulación de la prueba pericial, el problema surgió, de nuevo, con motivo de la reforma operada en la LECR en el año 2002. Así el art. 788.2 LECR otorga naturaleza de prueba documental a los informes emitidos por laboratorios oficiales para el caso sólo de materia de estupefacientes y aunque la Ley no dice nada de los informes de ADN, ante el silencio, se debe entender que estamos ante una prueba de naturaleza pericia.

33 Se trata de los contenidos la Sentencia del Tribunal Supremo Federal de los Estados Unidos, Daubert v. Merrell Dow Pharmaceuticals, Inc. (509 U.S. 579, 113 (1993). REDMAYNE, M. “Admissibility Standards for Scientific Evidence”, In I. Madellin (ed.), Admissibility Evidence in Criminal Cases, Collected Papers, 1998. Del mismo autor Expert Evidence and Criminal Justice, New York: Oxford University Press, 2001. Y que la doctrina más especializada estudia, vid., por todos, BACHMAIER WINTER, Lorena. "Dos modelos de prueba pericial en el derecho comparado: Estados Unidos de América y Alemania. Jueces para la democracia, $n^{\circ} 66$, noviembre 2009, pp. 118-137. También, DOLZ LAGO, Manuel Jesús. La prueba de ADN a través de la jurisprudencia. Una visión práctica y crítica. La Ley - Wolters Kluwer. Madrid: 2016, p. 41. GÓMEZ COLOMER, Juan Luis. "La prueba científica, motor de cambios esenciales en el proceso penal moderno", Garantismo frente a eficacia, justicia frente a seguridad, p. 5. Disponible: http://perso.unifr.ch/derechopenal/assets/files/articulos/a_20170408_01.pdf (Fecha acceso: 14/11/2019). 


\section{La aplicación de la Inteligencia Artificial en el proceso penal}

El interés por la utilización de tecnologías de inteligencia artificial en el proceso judicial lo podemos relacionar con la necesidad de mejorar su eficacia. No obstante, somos conscientes de que se trata de una cuestión no exenta de polémica, no sólo por los peligros que conlleva la aplicación de inteligencia artificial en el proceso sino, también, por pretender que la utilización de la inteligencia artificial tenga por finalidad que el proceso sea más eficaz. Centrándonos en esta última cuestión, en el término "eficacia"; cuando utilizamos ese término referido al proceso, hablando de eficacia en el proceso y no, en esencia, de las garantías procesales, se tiene la sensación de cierta connotación economicista; parece que, de alguna manera estamos reduciendo a lo privado el sistema judicial penal que tiene, por naturaleza, carácter público. Nada más lejos de la realidad, cuando un proceso es ineficaz, es un proceso inútil que no cumple o impide la función constitucional encomendada al Poder Judicial ni garantiza, tampoco, el derecho constitucional a la jurisdicción que tienen los ciudadanos ${ }^{35}$.

Es por esta razón que el estudio de la posibilidad de aplicar inteligencia artificial en el proceso y, en este caso, en el proceso penal, aunque, sin duda, controvertido, no puede por este motivo dejarse de estudiar, quizá aunque sólo sea para llegar a la conclusión de que su uso en el proceso es inviable. La doctrina procesalista se ha pronunciado recientemente sobre esta cuestión ${ }^{36}$ y se muestra reticente a una aplicación

35 MONTERO AROCA, Juan. DERECHO JURISDICCIONAL I. Parte General. ed. $27^{\mathrm{a}}$. Valencia: Tirant lo Blanch, 2018, p. 35, cuando explica la evolución científica del Derecho Jurisdiccional, expone "Cuando se habla de eficacia de la eficacia del proceso se está en realidad cuestionando la eficacia del Poder Judicial, y de ahí la gravedad de unas leyes procesales no adecuadas para hacer frente a las necesidades reales de la sociedad."

36 Véase DE HOYOS SANCHO, Monserrat. "El libro blanco sobre inteligencia artificial de la Comisión Europea: reflexiones desde las garantías esenciales del proceso penal como "sector de riesgo". Revista española de derecho europeo, $\mathrm{n}^{\circ} 76,2020$. MARTÍN DIZ, Fernando. Herramientas de inteligencia artificial y adecuación en el ámbito del proceso judicial. In: BUJOSA VADELL, Lorenzo M. (dir.); GONZÁLEZ PULIDO, Irene (coord..); REIFARTH MUÑOZ, Walter (coord..). Derecho procesal: retos y transformaciones. Madrid: Atelier, 2021. MARTÍN DIZ, Fernando. Inteligencia artificial y derecho procesal: Luces, 
generalizada en el proceso y, más aún, si se trata del proceso penal, por la especial configuración de éste y los derechos que en él se garantizan, debido a los múltiples riesgos que su uso puede suponer para los derechos y garantías fundamentales procesales, sobre todo, en el caso de aplicar inteligencia artificial para la toma de decisiones judiciales, sustituyendo la decisión judicial ${ }^{37}$.

No obstante, dentro de las tecnologías que utilizan inteligencia artificial existe una que ha evolucionado de una manera sorprendente estos últimos años $y$, que entendemos, merece la pena analizar por si, en un futuro no muy lejano, puede servir de ayuda en la fase probatoria del proceso penal: hablamos de tecnología de reconocimiento facial ${ }^{38}$.

\subsection{TECNOLOGÍA DE RECONOCIMIENTO FACIAL}

La tecnología del reconocimiento facial, que utiliza inteligencia artificial en su desarrollo, posibilita el reconocimiento de los rasgos faciales de una persona. Se trata de analizar si esta tecnología puede resultar de utilidad en el proceso penal, en concreto en el desarrollo de la actividad probatoria. El tratamiento que esta tecnología puede

sombras y cábalas en clave de derechos fundamentales. In: MORENO CATENA, Victor Manuel (dir.); ROMERO PRADAS, María Isabel (dir.); LARO GONZÁLEZ, María Elena (ed. Lit.). Nuevos postulados de la cooperación judicial en la Unión Europea. Libro homenaje a la Prof. ${ }^{a}$ Isabel González Cano. Valencia: Tirant lo Blanch, 2021. BUENO DE MATA, Federico, Macrodatos, inteligencia artificial y proceso: luces y sombras. Revista General de Derecho Proceal, $\mathrm{n}^{\circ}$ 51, 2020.

37 Sobre los riesgos de la inteligencia artificial en el proceso penal, GÓMEZ COLOMER, Juan Luis. La contracción del Derecho Procesal Penal. Valencia: Tirant lo Blanch, 2020, pp. 421-437. También BARONA VILAR, Silvia. Inteligencia artificial o la algoritmización de la vida y de la justicia: ¿solución o problema? Revista Boliviana de Derecho, n. 28, 2019, pp. 18-49. NIEVA FENOLL, Jordi. Inteligencia artificial y proceso judicial. Madrid: Marcial Pons, 2018.

38 Se ocupa del estudio de esta tecnología y su impacto en el ámbito de la Justicia BEIRO MAGÁN, José Manuel. Automatización e inteligencia artificial: por qué debe generalizarse su implantación en el sector justicia español, $P O-$ nencia presentada en Granada el 12 de marzo de 2020 en las Jornadas sobre "La organización de la OJ y la OF en la transformación tecnológica. Aplicaciones de inteligencia artificial”. 
realizar de los metadatos ${ }^{39}$ de las grabaciones y registros audiovisuales judiciales puede tener relevancia en el desarrollo de la actividad probatoria. Nos estamos refiriendo a la comunicación no verbal que se presenta en los actos judiciales como la que ocurre en la vista de un juicio oral o en una declaración judicial y que resulta imposible de verbalizar o transcribir; esto supone que todos esos datos, fruto de la comunicación no verbal, que pasan desapercibidos para el ser humano puedan llegar, si se interpretan con el reconocimiento facial, a ser valorados también por el juez como prueba. Esta tecnología de reconocimiento facial puede servir, también, a los efectos de averiguar la identidad del presunto autor del hecho o, incluso, en el caso de identificación de testigos, peritos o, incluso, requisitoriados. Sin duda, va a ser la primera de las funciones de la tecnología del reconocimiento facial, que hemos señalado, la que presente mayor problemática, desde el punto de vista procesal, porque el tratamiento de los metadatos de las declaraciones o interrogatorios va a aportar un valor añadido a la declaración o interrogatorio judicial que se preste. Es decir, más allá de las palabras la comunicación no verbal del testigo o de la parte que preste declaración podría formar parte de la declaración y servir a los efectos de verificar la credibilidad, por ejemplo, del testigo.

Uno de los grandes problemas del uso de esta tecnología es que afecta a derechos especialmente protegidos como es el derecho fundamental a la intimidad y, en concreto, al derecho a la protección de los datos personales. Precisamente, el nuevo Reglamento General de Protección de Datos (RGPD) ${ }^{40}$, consciente de la necesidad de protección de este derecho, realiza una modificación en la calificación jurídica de los

39 Los metadatos son datos que describen el contenido de los archivos o la información de otros datos. "¿Qué son los metadatos y cuál es su utilidad?", PowerData, marzo 19, 2016. Disponible: https://blog.powerdata.es/el-valor-de-la-gestion-de-datos/que-son-los-metadatos-y-cuales-su-utilidad

40 Reglamento (UE) 2016/679 del Parlamento Europeo y del Consejo, de 27 de abril de 2016, relativo a la protección de las personas físicas en lo que respecta al tratamiento de datos personales y a la libre circulación de estos datos y por el que se deroga la Directiva 95/46/CE (Reglamento general de protección de datos). DOUE 4/5/2016. 
datos biométricos ${ }^{41}$, que pasa a considerarlos datos de categoría especial, en virtud del art. 9.1 RGPD. El precepto hace referencia, en especial, a los datos biométricos que están dirigidos a identificar de manera inequívoca a una persona física. Además, y con el motivo de otorgar protección a la persona, garantizando su dignidad, el RGPD limita el uso de la tecnología de reconocimiento facial y lo hace impidiendo su tratamiento, de forma general, con las excepciones que se contemplan en el apartado 2 del art. 9 RGPD, entre las que se encuentran las que están relacionadas con la actividad judicial o policial o de seguridad pública ${ }^{42}$.

Otro de los problemas jurídicos que presenta esta tecnología de reconocimiento facial es el de los sesgos algorítmicos, a los que nos hemos referido en epígrafe anterior, y que provoca afectaciones en determinados derechos fundamentales, siendo una de las más relevantes la vulneración del principio de igualdad, pero también afecta a derechos procesales fundamentales ${ }^{43}$. El problema de los sesgos algorítmicos en el uso de esta tecnología está relacionado, sobre todo, con la utilización de los algoritmos de aprendizaje automático (los que entendemos, estrictamente, por inteligencia artificial). Estos algoritmos están diseñados para tratar los datos como lo haría una persona y al combinarlos con la tecnología de reconocimiento facial se obtienen unos resultados más eficientes, más complejos. El problema es la evolución de estos algoritmos, que

${ }^{41}$ Según el art. 4 RGPD, los datos biométricos se definen como aquellos "datos personales obtenidos a partir de un tratamiento técnico específico, relativos a las características físicas, fisiológicas o conductuales de una persona física que permitan o confirmen la identificación única de dicha persona, como imágenes faciales o datos dactiloscópicos".

42 Entre las excepciones a las que alude el art. 9.2 Reglamento General de Protección de Datos, nos encontraríamos, fundamentalmente la existencia del consentimiento explícito del interesado, el cumplimiento de obligaciones o ejercicio de derechos por parte del responsable del tratamiento, la protección de intereses vitales del titular de los datos, o la presencia de un interés público, entre otros.

43 Nos referimos al principio de imparcialidad judicial, así como a la igualdad de las partes en el proceso, como o el derecho fundamental de audiencia o defensa, que tratamos en DE MIGUEL BERIAIN, Iñigo; PÉREZ ESTRADA, M. Josune; La inteligencia artificial en el proceso penal español: un análisis de su admisibilidad sobre la base de los derechos fundamentales implicados, op. cit., p. 550. 
no puede ser explicada de forma lógica, lo que provoca opacidad en su proceso de uso ${ }^{44}$.

La preocupación de la Unión Europea por la posible vulneración de los derechos fundamentales con este tipo de tecnología de reconocimiento facial en la que se utilizan datos biométricos ha quedado patente con Informe de la Agencia Europea de Derechos Fundamentales (FRA), emitido en 2019, sobre Tecnología de reconocimiento facial: consideraciones de derechos fundamentales en el contexto de la aplicación de la ley ${ }^{45}$. Junto con éste, el informe de la Comisión Europea White paper On Artificial Intelligence-A European approach to excellence and trust, muestra que la regulación de la implantación de tecnología de reconocimiento facial contendrá las restricciones necesarias para proteger los derechos fundamentales, siendo los más afectados por esta tecnología el derecho a la privacidad y la protección de los datos personales ${ }^{46}$. Precisamente y sólo de esta manera, con una protección al más alto nivel de los derechos fundamentales especialmente afectados podremos garantizar un futuro uso adecuado de esta tecnología en el proceso judicial.

44 El informe "The Perpetual Line Up" del Georgetown Law Center on Privacy and Technology incorpora determinadas recomendaciones a los poderes públicos de los Estados Unidos entre las que destaca una mejor regulación legal, así como mayor esfuerzo de transparencia pública de los procesos relativos a la obtención y gestión de los datos. Disponible: https://www.law.georgetown.edu/privacy-technology-center/publications/the-perpetual-line-up/ Fecha acceso: 09/09/2020.

45 Facial recognition technology: fundamental rights considerations in the context of law enforcement. El documento analiza las implicaciones de derechos fundamentales de confiar en FRT en vivo (comparación de imágenes obtenidas de cámaras de video (CCTV) con imágenes en bases de datos), centrándose en su uso para fines de aplicación de la ley y gestión de fronteras. Disponible: https://fra.europa.eu/en/publication/2019/facial-recognition-technology-fundamental-rights-considerations-context-law Fecha acceso: 09/09/2020.

BEIRO MAGÁN, José Manuel. Automatización e inteligencia artificial: por qué debe generalizarse su implantación en el sector justicia español, op. cit., nos recuerda “... el polémico proyecto iBorderCtrl, que mediante el uso de tecnología de reconocimiento biométrico permite al personal de fronteras recopilar dichos datos de la persona migrante a efectos de incorporarlos a la base correspondiente, así como detectar si es o no ciudadano comunitario en el momento de ingresar, o emitir una valoración sobre si dice o no la verdad al personal de migraciones." 


\subsection{El sentido de la Inteligencia Artificial como prueba científica en EL PROCESO PENAL}

Cabe plantearse si estas herramientas inteligentes pueden constituir una forma de prueba científica como lo es el análisis del ADN, o, por el contrario, estamos muy lejos de poder otorgarles esta categoría. Al contrario que en otros países, no existe en España un procedimiento estandarizado que permita dilucidar si una determinada técnica puede considerarse prueba científica o qué parámetros tiene que cumplir para llegar a serlo ${ }^{47}$. La determinación de la cientificidad de la inteligencia artificial a los efectos de considerarla como prueba es un tema que deberá abordarse a medida que se perfeccione la técnica o se generalice su uso. Como nos recuerda Gómez Colomer los criterios de cientificidad que sirven para considerar que una prueba tiene la categoría de científica en nada tienen que ver con la admisibilidad de la prueba que se rige por el criterio de la pertinencia judicial. Ni tampoco influyen sobre la valoración de la prueba que realice el juez en virtud del principio de libre valoración de la prueba que rige en el proceso penal ${ }^{48}$.

En esta cuestión habrá que valorar si dichas herramientas de inteligencia artificial deben tener un grado de fiabilidad absoluto, como ocurre con la prueba del ADN, o, por el contrario, es suficiente si suponen una mejora de la veracidad o certeza que otorgan los medios de prueba tradicionales y a los que no se cuestiona su uso ${ }^{49}$.

47 En EE. UU. se utiliza el denominado Test de Daubert, que se basa en el análisis de una serie de parámetros, a fin de considerar que una técnica novedosa reúne un mínimo consenso científico necesario para su empleo en el ámbito forense. Sobre esta cuestión, véase, ALCOCEBA GIL, J. Manuel. Los estándares de cientificidad como criterio de admisibilidad de la prueba científica. Revista Brasileira de Direito Processual Penal, vol. 4, n. 1, p. 215-242, jan./abr. 2018.

Recordemos que Recuerda estos conceptos GÓMEZ COLOMER, Juan Luis La prueba científica, motor de cambios esenciales en el proceso penal moderno, op. cit., pp. 5 y 15.

49 VILLAMARÍN LÓPEZ, María Luisa. Neurociencia y detección de la verdad y del engaño en el proceso penal. El uso del escáner cerebral (fMRI) y del brainfingerprinting (P300.,Madrid: , 2014, cuando escribe que "lo que nos parece llamativo es el altísimo nivel de exigencia en cuanto a la fiabilidad de estas técnicas, que, en general, superan lo exigido por la comunidad científica para su reconocimiento, mientras se siguen admitiendo sin dudar medios de investigación o de prueba bastante o muy endebles que, al menos por el momento, 
Una de las cuestiones relevantes para sostener la posibilidad de aplicación en el proceso de un sistema de inteligencia artificial es la verificación de los algoritmos que contienen. Esta verificación de un algoritmo debe prestar atención tanto a la posibilidad de que presente sesgos que discriminen a unas personas frente a otras, de acuerdo con los intereses o las inclinaciones de los programadores, como a su grado de fiabilidad predictiva, lo que muchas veces dependerá de la propia calidad de los datos utilizados en su confección ${ }^{50}$. Por esto mismo, parece evidente que, como ha señalado el Parlamento Europeo, serán necesarias "evaluaciones periódicas sobre la representatividad de los conjuntos de datos, así como examinar la exactitud e importancia de las predicciones" ${ }^{51}$. De esta manera, antes de tomar una decisión sobre la posibilidad de utilizar estos mecanismos en nuestro sistema jurídico es necesario resolver previamente la cuestión de cómo regular los mecanismos que garantizarán una adecuada auditoría de su funcionamiento ${ }^{52}$. Sin embargo, esta necesidad

pueden ser confrontados con estos nuevos procedimientos". También NIEVA FENOLL, Jordi. Neurociencia y Juicio Jurisdiccional: Pasado y presente. ¿Futuro?, La ciencia jurisdiccional: novedad y tradición. Madrid: Marcial Pons, 2016. Y del mismo autor, La discutible utilidad de los interrogatorios de partes y testigos., Madrid: Diario La Ley, n. 9672, 2020.

50 Como señala el Considerando M de la Resolución del Parlamento Europeo, de 14 de marzo de 2017, "debe hacerse una distinción entre cantidad y calidad de los datos a fin de facilitar la utilización eficaz de los macrodatos (algoritmos y otras herramientas analíticas); y que los datos y/o los procedimientos de baja calidad en los que se basan los procesos de toma de decisiones y las herramientas analíticas podrían dar lugar a algoritmos sesgados, correlaciones falsas, errores, una subestimación de las repercusiones éticas, sociales y legales, el riesgo de utilización de los datos con fines discriminatorios o fraudulentos y la marginación del papel de los seres humanos en esos procesos, lo que puede traducirse en procedimientos deficientes de toma de decisiones con repercusiones negativas en las vidas y oportunidades de los ciudadanos, en particular los grupos marginalizados, así como generar un impacto negativo en las sociedades y empresas". BOYD Y CRAWFORD, sostiene que los números no hablan por sí mismo; las afirmaciones de objetividad y precisión son engañosas dado que todos los investigadores son intérpretes de datos y siempre hay un proceso de "limpieza de datos" inherentemente subjetivo. BOYD, D.; CRAWFORD, K. Six Provocations for Big Data, Symposium on the Dynamics of the Internet and Society, (September 21, 2011): A Decade in Internet Time, op. cit.

51 Resolución del Parlamento Europeo, de 14 de marzo de 2017, Considerando 20.

52 Algunas sugerencias figuran en el "discussion paper" del UK ICO, que señala que "Las técnicas de auditoría pueden utilizarse para identificar los factores 
que no es fácil de realizar porque muchos de los algoritmos que contiene los sistemas de inteligencia artificial son, en sí mismos, opacos ${ }^{53}$.

\section{Conclusiones}

A modo de conclusión, quizá no se debiera exigir que para su uso en el proceso estas técnicas de inteligencia artificial tengan un grado de fiabilidad absoluto, pues tampoco gozan de ese grado de certeza otros medios de prueba, pero, teniendo en cuenta el riesgo que conllevan sí, necesariamente, debe vigilarse un total encaje en el sistema de protección de los derechos fundamentales y garantías procesales. Puesto que el error en la fuente de datos debido al uso de datos no fiables o de baja calidad o la existencia de sesgos en el propio algoritmo o, incluso, en las interpretaciones de las conclusiones obtenidas afectaría, en el caso de que se utilizase en el proceso al desarrollo de la convicción judicial. Además, la lógica del algoritmo o su diseño debe ser comprensible para las partes del proceso de manera

que influyen en una decisión algorítmica. Los sistemas de visualización interactiva pueden ayudar a los individuos a entender por qué se hizo una recomendación concreta y darles control sobre futuras recomendaciones. Los comités de ética pueden utilizarse para ayudar a dar forma y mejorar la transparencia del desarrollo de algoritmos de aprendizaje automático. Debemos, en suma, utilizar sistemas de supervisión que combinen garanticen la transparencia de los algoritmos empleando a la vez un enfoque técnico y organizativo". UK Information Commissioner's Office (ICO), Big data, artificial intelligence, machine learning and data protection, 2017, p. 86. Disponible: https://ico.org.uk/media/for-organisations/documents/2013559/big-dataai-ml-and-data-protection.pdf Fecha acceso: 13/11/2020

Como escribe COTINO HUESO, "Big data e inteligencia artificial. Una aproximación a su tratamiento jurídico desde los derechos fundamentales", op. cit., 139 “...entiendo que este tipo de garantías frente a la discriminación van a tener que combinarse con el reconocimiento de fuertes facultades de acceso y conocimiento de los algoritmos y los grandes datos que se manejan por parte de sectores especializados, tanto públicos como de la sociedad civil (reguladores, académicos, industria, asociaciones de consumidores, etc. (...).” Y dicha transparencia ha de venir acompañada asimismo con el reconocimiento de fuertes potestades de control a autoridades independientes respecto de los "data brokers" que son claves en el sector. Sin perjuicio del control social por la sociedad civil especializada, entiendo que son idóneas las autoridades independientes cercanas al sector de las nuevas tecnologías, como lo son las autoridades de protección de datos y transparencia. EU, AEPD-ISMS, 2017, Código de buenas prácticas en protección de datos para proyectos de Big Data”, op. cit. págs. 26 y ss. 
que se garantice el derecho de defensa o audiencia. Debemos recordar que la valoración de la prueba en el proceso penal es función jurisdiccional por lo que le corresponde al juez realizarla, pero, entendemos que es posible el uso de inteligencia artificial en el proceso penal, bien como medio de prueba $o$ utilizada junto con los medios de prueba tradicionales para que puedan éstos ser más efectivos y así ayudar al juez en la valoración judicial de la prueba.

Entendemos que las decisiones generadas por inteligencia artificial sólo se deben utilizar como una herramienta más en la ayuda de la toma de decisión pero sin olvidar la capacidad humana crítica, de interpretación de los resultados, que no poseen las máquinas, ni ninguna, por ahora, inteligencia artificial ${ }^{54}$. Esta posición se aparta del criterio de que el hecho de apartarse de una decisión automática generada por una aplicación algorítmica era algo inverosímil por otorgarle, de alguna manera, cierta presunción de veracidad. Esta idea es fundamental porque argumenta la tesis de que el uso de inteligencia artificial no debe sustituir la toma de decisiones judicial sino servir de ayuda, en este caso, en la valoración de la prueba. ${ }^{55}$

\section{REFERENCIAS BIBLIOGRÁFICAS}

ALCOCEBA GIL, J. Manuel. Los estándares de cientificidad como criterio de admisibilidad de la prueba científica., Revista Brasileira de Direito Processual Penal, vol. 4, n. 1, p. 215-242, jan./abr. 2018. http://dx.doi.org/10.22197/rbdpp.v4i1.120

54 Disciplinas como la neurociencia vienen demostrando que la "inteligencia intuitiva o emocional", propia de los seres humanos, y las decisiones con base en la intuición no son irracionales, sino que ayudan en la toma de decisiones complejas incapaz de percibirlas la inteligencia artificial pero imprescindibles en la adopción de cualquier tipo de decisión. MARTÍNEZ MARTÍNEZ, Ricard. Cuestiones de ética jurídica al abordar proyectos de Big Data. El contexto del Reglamento general de protección de datos, Dilemata, $\mathrm{n}^{\circ} .24,2017$, p. 154.

55 Este uso del resultado de los datos desde un punto de vista crítico nos da la posibilidad de que podamos apartarnos de la decisión automática. Precisamente, el Reglamento General de Protección de Datos, prohíbe, de manera general, las decisiones que se basan sólo en el tratamiento automatizado de los datos y la elaboración de perfiles que puedan tener efectos jurídicos, sin que intervenga ninguna persona en la decisión. Así el art. 22.1 Reglamento General de Protección de Datos establece: “Todo interesado tendrá derecho a no ser objeto de una decisión basada únicamente en el tratamiento automatizado, incluida la elaboración de perfiles, que produzca efectos jurídicos en él o le afecte significativamente de modo similar." 
ASENSIO MELLADO, José María. “La prueba penal”. In: ASENSIO MELLADO, José María (dir.); FUENTES SORIANO, Olga (coord.). Derecho procesal penal (dir., coord.), Valencia:Tirant lo Blanch, 2020.

BACHMAIER WINTER, Lorena. "Dos modelos de prueba pericial en el derecho comparado: Estados Unidos de América y Alemania. Jueces para la democracia, $\mathrm{n}^{\mathrm{o}}$ 66, noviembre 2009, pp. 118-137.

BARONA VILAR, Silvia. Cuarta revolución industrial (4.0.) o ciberindustria en el proceso penal: revolución digital, inteligencia artificial y el camino hacia la robotización de la justicia. Revista Jurídica Digital UANDES, Vol. 3, n. 1, Chile, 2019. https://doi.org/10.24822/rjduandes.0301.1

BARONA VILAR, Silvia. Inteligencia artificial o la algoritmización de la vida y de la justicia: ¿solución o problema? Revista Boliviana de Derecho, n. 28, 2019.

BEIRO MAGÁN, José Manuel. Automatización e inteligencia artificial: por qué debe generalizarse su implantación en el sector justicia español, Ponencia presentada en Granada el 12 de marzo de 2020 en las Jornadas sobre "La organización de la OJ y la OF en la transformación tecnológica. Aplicaciones de inteligencia artificial". BONAZZI, M., "Reconstructing man? The power of converging technologies”, 2006. http://cordis.europa.eu/wire/index.cfm?fuseaction=article. Detail\&rcn=11117 (fecha acceso: 20/05/2021).

BOYD, David; CRAWFORD, Kate. Critical questions for Big Data. Provocations for a cultural, technological and scholarly phenomenon., Journal Information, Communication and Society, n. 5, vol. 15, 2012. https://doi.org/10.1080/1369118x.2012.678878 BUENO DE MATA, Federico, Macrodatos, inteligencia artificail y proceso: luces y sombras. Revista General de Derecho Proceal, $\mathrm{n}^{\circ}$ 51, 2020.

BYUNG-CHUL, Han. La sociedad de la transparencia. Barcelona: Herder, 2013. http://dx.doi.org/10.15446/ideasyvalores.v63n156.47043

COGLIANESE, Cary and LEHR, David. Regulating by Robot: Administrative Decision Making in the Machine-Learning Era. Georgetown Law Journal, Forthcoming; U of Penn: Inst for Law \& Econ Research Paper, n. 17-8, febrero 2017.

COTINO HUESO, Lorenzo. Big data e inteligencia artificial. Una aproximación a su tratamiento jurídico desde los derechos fundamentales. Madrid: Dilemata, 2017.

DE HOYOS SANCHO, Monserrat. "El libro blanco sobre inteligencia artificial de la Comisión Europea: reflexiones desde las garantías esenciales del proceso penal como "sector de riesgo". Revista española de derecho europeo, n 76, 2020. https:// doi.org/10.37417/REDE/num76_2020_534

DE MIGUEL BERIAIN, Iñigo; PÉREZ ESTRADA, M. Josune. La inteligencia artificial en el proceso penal español: un análisis de su admisibilidad sobre la base de 
los derechos fundamentales implicados, Revista de derecho UNED (RDUNED), $\mathrm{n}$ 25, Madrid, 2019, pp. 531-561. https://doi.org/10.5944/rduned.25.2019.27013. Disponible: http://perso.unifr.ch/derechopenal/assets/files/articulos/a_20170408_01.pdf

DOLZ LAGO, Manuel Jesús. La prueba de ADN a través de la jurisprudencia. Una visión práctica y crítica. La Ley - Wolters Kluwer. Madrid: 2016.

EDWARS, Llilian, VEALE, Michael. Slave to the algorithm? Why a "Right to an explanation" is probably not the remedy you are looking for. Drurham: Duke Law \& Technology Review, 2017. http://dx.doi.org/10.2139/ssrn.2972855

ETXEBERRIA GURIDI, J.F., El análisis de ADN y su aplicación al proceso penal, Ed. Comares, Granada, 2000.

FERRER BELTRÁN, Jordi. Prueba y verdad en el derecho. Madrid: Marcial Pons, 2005.

GASCÓN ABELLÁN, Marina. Validez y valor de las pruebas científicas: la prueba del ADN. Universidad de Castilla- La Mancha, 2015.

GÓMEZ COLOMER, Juan Luis. "La prueba científica, motor de cambios esenciales en el proceso penal moderno", Garantismo frente a eficacia, justicia frente a seguridad.

GÓMEZ COLOMER, Juan Luis. La contracción del Derecho Procesal Penal. Valencia: Tirant lo Blanch, 2020.

GÓMEZ COLOMER, Juan Luis. Los retos del proceso penal ante las nuevas pruebas que requieren tecnología avanzada: $\mathrm{El}$ análisis de ADN.” In: GÓMEZ COLOMER, Juan Luis (coord.), La prueba de ADN en el proceso penal. Valencia: Tirant lo Blanch, 2014.

GUZMÁN FLUJA, Vicente C., Sobre la aplicación de la inteligencia artificial a la solución de conflictos (Reflexiones acerca de una transformación tan apasionante como compleja. In: BARONA VILAR, Silvia (ed.). Justicia Civil y Penal en la era global, Valencia: Tirant lo Blanch, 2017.

HARTUNG, Markus, BUES, Micha-Manuel y HALBLEIB, Gernot, AAVV, Legal Tech. A Practitioner's Guide, Germany: Beck/Hart, 2018.

LACRUZ MANTECÓN, Miguel L., "Cibernética y Derecho Europeo: zuna inteligencia robótica?”. Madrid: Diario La Ley, n. 9376, Sección Doctrina, 2019.

LEHR, David \& OHM, Paul. Playing with the Data: What Legal Scholars Should Learn About Machine Learning. Georgetown Law Journal, 2017.

MAGRO SERVET, Vicente. La aplicación de la inteligencia artificial en la Administración de Justicia. Madrid: Diario La Ley, n.. 9268, Sección Doctrina, 2018.

MARTÍN DIZ, Fernando. Herramientas de inteligencia artificial y adecuación en el ámbito del proceso judicial. In: BUJOSA VADELL, Lorenzo M. (dir.); GONZÁLEZ 
PULIDO, Irene (coord..); REIFARTH MUÑOZ, Walter (coord..). Derecho procesal: retos y transformaciones. Madrid: Atelier, 2021.

MARTÍN DIZ, Fernando. Inteligencia artificial y derecho procesal: Luces, sombras y cábalas en clave de derechos fundamentales. In: MORENO CATENA, Victor Manuel (dir.); ROMERO PRADAS, María Isabel (dir.); LARO GONZÁLEZ, María Elena (ed. Lit.). Nuevos postulados de la cooperación judicial en la Unión Europea. Libro homenaje a la Prof. ${ }^{a}$ Isabel González Cano. Valencia: Tirant lo Blanch, 2021.

MARTÍNEZ MARTÍNEZ, Ricard. Cuestiones de ética jurídica al abordar proyectos de Big Data. El contexto del Reglamento general de protección de datos, Dilemata, no. 24, 2017, p. 154.

MILÓN BELTRÁN, Noemí. "Retos para la privacidad en la Era Digital. Análisis económico y filosófico político del capitalismo contemporáneo”. Sociología y Tecnociencia. Revista digital de sociología del sistema tecnocientífico, vol. 2, n.. 5, 2015.

MITTELSTADT, Brent Daniel; ALLO, Patrick; TADDEO, Maria Rosaria; WATCHER, Sandra, FLORIDI, Luciano,.The ethics of algorithms: Mapping the debate" Oxford Internet Institute, Diciembre, 2016.

MONTERO AROCA, Juan. DERECHO JURISDICCIONAL I. Parte General. ed. 27a . Valencia: Tirant lo Blanch, 2018.

MORENTE PARRA, Vanesa. Big Data o el arte de analizar datos masivos. Una reflexión crítica desde los derechos fundamentales. Derechos y Libertades, n. 41, Época II, junio 2019.

MÜULLER, Vincent C., BOSTROM, Nick. Future Progress in Artificial Intelligence: A Survey of Expert Opinion. In: MÜLLER, Vincent C. (ed.). Fundamental Issues of Artificial Intelligence. Berlin: Springer, 2014.

NIEVA FENOLL, Jordi. La discutible utilidad de los interrogatorios de partes y testigos., Madrid: Diario La Ley, n. 9672, 2020. https://doi.org/10.4067/ s0718-00122020000300157

NIEVA FENOLL, Jordi. Inteligencia artificial y proceso judicial. Madrid: Marcial Pons, 2018.

NIEVA FENOLL, Jordi. Neurociencia y Juicio Jurisdiccional: Pasado y presente. ¿Futuro?, La ciencia jurisdiccional: novedad y tradición. Madrid: Marcial Pons, 2016.

O’NEIL, Cathy Armas de destrucción matemática., Madrid: Capitán Swing, 2017. PASTOR GARCÍA, Luis Miguel; GARCÍA CUADRADO, José Ángel. Modernity and postmodernity in the genesis of posthumanism, Cuadernos de bioética, (Ejemplar dedicado a: Posthumano, más que humano), Vol. 25, nº 85, 2014.

PÉREZ ESTRADA, M. Josune. El uso de algoritmos en el proceso penal y el derecho a un proceso con todas las garantías. In: BARONA VILAR, Silvia (ed.). Claves de 
la justicia penal: feminización, inteligencia artificial, supranacionalidad y seguridad, Valencia: Tirant lo Blanch, 2019.

PÉREZ GIL, Julio. El conocimiento científico en el proceso civil. Ciencia y tecnología en tela de juicio. Valencia: Tirant lo Blanch, 2010.

PICÓ i JUNOY, Joan (dir.); ANDINO LÓPEZ, Juan Antonio (coord..); CERRATO GURI, Elisabet (coord.). La prueba periscial a examen. Madrid: J. M. Bosch Editor 2020.

QUADRA-SALCEDO FERNÁNDEZ DEL CASTILLO, Tomás, Derechos fundamentales, democracia y mercado en la edad digital, Derecho Digital e Innovación, núm. 1, Madrid, enero-marzo 2019.

QUADRA-SALCEDO FERNÁNDEZ DEL CASTILLO, Tomás. Retos, riesgos y oportunidades de la Sociedad digital", In: QUADRA-SALCEDO y PIÑAR MAÑAS (dir.), Sociedad Digital y Derecho. Madrid: BOE. Ministerio de Industria, Comercio y Turismo y Red.es, 2018.

REDMAYNE, M. "Admissibility Standards for Scientific Evidence”, In: I. Madellin (ed.), Admissibility Evidence in Criminal Cases, Collected Papers 1998.

REDMAYNE, M. Expert Evidence and Criminal Justice. New York: Oxford University Press 2001.

SURDEN, Harry. Values Embedded in Legal Artificial Intelligence. Documento de investigación de estudios legales de la U. of Colorado, n. 17-17, 2017.

TARUFFO, Michele. La prueba de los hechos (trad. Ferrer Beltrán). Madrid: Trotta, 2002.

VILLAMARÍN LÓPEZ, María Luisa. Neurociencia y detección de la verdad y del engaño en el proceso penal. El uso del escáner cerebral ( $f M R I)$ y del brainfingerprinting (P300), Madrid: 2014.

\section{Additional information and author's declarations (scientific integrity)}

Acknowledgement: Este trabajo se ha realizado en el contexto de las actividades que realiza el Grupo de investigación consolidado "Derechos Fundamentales y Unión Europea. Especial referencia al Espacio de Libertad, Seguridad y Justicia de la Unión Europea" (GIC IT-673-13, 2019-2021), así como dentro del contexto del Proyecto H2020-SWAFS17/01. 
Conflict of interest declaration: the author confirms that there are no conflicts of interest in conducting this research and writing this article.

Declaration of authorship: all and only researchers who comply the authorship requirements of this article are listed as authors; all coauthors are fully responsible for this work in its entirety.

Declaration of originality: the author assures that the text here published has not been previously published in any other resource and that future republication will only take place with the express indication of the reference of this original publication; he also attests that there is no third party plagiarism or self-plagiarism.

\section{Editorial process dates}

(http://www.ibraspp.com.br/revista/index.php/RBDPP/about/editorialPolicies)

- Submission: 21/12/2020

- Desk review and plagiarism check: 08/01/2021

- Resubmission and transfer to V7N2: 23/02/2021

- Review 1: 04/03/2021

- Review 2: 15/03/2021

- Review 3: 24/03/2021

- Preliminary editorial decision: $16 / 05 / 2021$

- Correction round return: 16/06/2021

- Final editorial decision: 19/06/2021

\section{HOW TO CITE (ABNT BRAZIL):}

PÉREZ ESTRADA, Miren Josune. La inteligencia artificial como prueba científica en el proceso penal español. Revista Brasileira de Direito Processual Penal, vol. 7, n. 2, p. 1385-1410, mai./ago. 2021. https://doi.org/10.22197/rbdpp.v7i2.505

\section{Editorial team}

- Editor-in-chief: 1 (VGV)

- Reviewers: 3 